\title{
Managing hemophilia: the role of mobile technology
}

This article was published in the following Dove Press journal:

Smart Homecare Technology and TeleHealth

6 May 2014

Number of times this article has been viewed

\author{
Kate Khair' \\ Mike Holland ${ }^{2}$ \\ 'Haemophilia Centre, Great \\ Ormond Street Hospital for \\ Children NHS Foundation Trust, \\ London, ${ }^{2} \mathrm{Haemnet}$ Ltd, London, UK
}

Correspondence: Kate Khair Haemophilia Centre, Great Ormond Street Hospital for Children NHS Foundation Trust, London WCIN 3JH, UK

Tel +442078298846

Email kate.khair@gosh.nhs.uk

\begin{abstract}
The ubiquity of mobile technology offers the potential for instantaneous and two-way transfer of information, as well as the potential for improving medical care delivery and extending it to those in countries and regions with a less developed medical infrastructure. This review considers the role of mobile health (mHealth) technology in managing hemophilia. This is a disease area in which good record-keeping is an essential component of home-based hemophilia care. Health care professionals contribute patients' data to national registries and databases and frequently interrogate those data for commercial and academic purposes. Only rarely are the data used to directly improve the care of the individual patient. Patient-focused apps designed to respond to an individual's personal data may offer the potential to empower patients to live healthier lifestyles, reinforcing the care provided by health care professionals and offering a mechanism for peer-support and promoting adherence to an individualized management plan.
\end{abstract}

Keywords: mHealth, hemophilia, peer-support, adherence

\section{Introduction}

There is great enthusiasm for mobile technology as a means of delivering health care and promoting health. Recent years have witnessed enormous growth in wireless connectivity, and more than two-thirds of the world's population now owns a mobile phone. There are now more than 3.2 billion unique mobile users worldwide. ${ }^{1}$ This offers not only huge potential for instantaneous and two-way transfer of information, but also, at a time when medicine is increasingly individualized, the potential for improving care delivery and extending it to those in countries and regions with a less developed medical infrastructure.

The potential of mobile health (mHealth) is impressive. It could benefit ambulatory patients by allowing them to more easily and reliably self-diagnose acute symptoms, and by enabling those with long-term medical conditions to engage more fully in their own care. In theory, this could help to improve better control of long-term conditions and allow for more rapid diagnosis and treatment of common acute conditions, while also reducing unnecessary visits to hospital clinic offices and emergency departments, with a substantial impact on health care costs and an improvement in consumer convenience.

However, the actual evidence to support mHealth is, at best, preliminary. Researchers from the London School of Hygiene and Tropical Medicine recently conducted a systematic review of health interventions that have used mobile technology. ${ }^{2}$ They identified 75 trials of mobile technology interventions for patients with the aim of changing health behavior or improving disease management. Of these trials, 72 
had been conducted in high-income countries. Three trials showed clear benefits: two trials in the UK suggested that text-messaging based interventions could help people quit smoking, and one in Kenya suggested these mobile tools could help HIV-positive patients to take anti-retroviral medications more accurately. While the effects of mobile phone based interventions appeared promising in some other areas, further high quality trials are required to establish their precise effects.

A further review of 32 trials evaluated the effectiveness of mobile technology for health care providers or services. ${ }^{2}$ The reviewers found that while some interventions designed to provide support for health care providers improved aspects of clinical diagnosis and management, other interventions were less successful. For example, the use of mobile photos sometimes resulted in incorrect diagnoses compared with face-to-face diagnosis. Text-based appointment reminders were found to be better than no reminders but not better than reminders by phone or mail.

Given the potential benefits now afforded by mobile technology and the limited evidence to date, the authors of this review stress that additional testing is needed. However, technological developments will clearly always outstrip scientific efforts at evaluation, and the past five years have witnessed the rise of smartphones, tablet computers and other mobile devices, along with the use of software applications or "apps".

There is a growing market for health care-related apps: IMS Health estimates that in June 2013 there were 23,682 genuinely health care-related apps available for download from the US Apple iTunes app store. ${ }^{3}$ Of these, 16,275 apps were categorized as consumer/patient oriented and 7,407 apps as health care professional oriented. ${ }^{3}$ Most are developed and marketed with little regulatory oversight, which is clearly a concern, particularly for those with a diagnostic function and those that involve data collection. The US Food and Drug Administration (FDA) published a guidance document on mobile medical apps in September 2013 and indicated that where the intended use of an app is for diagnosis then it will be considered a device and subject to US FDA clearance. ${ }^{4}$ To date, the US FDA has approved about 100 mobile medical apps, most of which are focused on long-term condition management, including diabetes, asthma, and blood pressure management. ${ }^{5}$

For any app that collects, stores, or transmits information, there must be transparency and awareness of how data entered are to be used. It is essential that patient consent is obtained for use of that data. Any app that connects to an electronic or personal health record and that enables users to send and retrieve patient information between a mobile device and the record, must do so in a secure manner. It is also important that health care professionals accept their stewardship role for protecting the personal health data contained.

This review considers the role of mHealth in managing hemophilia, a disease area in which mHealth appears to have great potential.

\section{Haemophilia and mobile health}

Hemophilia is a rare, hereditary bleeding disorder caused by a deficiency of clotting factor VIII (FVIII) or factor IX (FIX) in the blood. These deficiencies arise as the result of mutations in the respective clotting factor genes.

Hemophilia occurs in all racial groups with an estimated frequency of approximately one in 10,000 births. Annual surveys conducted by the World Federation of Hemophilia suggest there are about 400,000 people with hemophilia worldwide. ${ }^{6}$ Despite the hereditary nature of the condition, around one third of cases occur in the absence of a known family history. ${ }^{7}$

The significance of hemophilia lies in the potential for spontaneous bleeding. The severity of bleeding correlates inversely with the clotting factor level; individuals with the lowest clotting factor levels are at high risk of repeated joint and muscle bleeds that can lead to progressive joint damage and long-term disability. Without treatment, there is also significant risk of intracranial bleeding and its severe complications, including permanent cerebral damage and death. Treatment by factor replacement therapy can stop or prevent bleeding episodes, reducing associated complications, improving quality of life, and normalizing life expectancy.

In developed countries, most people with hemophilia are able to self-administer their infusions at home, ${ }^{8}$ which has improved patients' quality of life by facilitating a primarily prophylactic approach to management. Self-infusions have resulted in bleed prevention, fewer hospitalizations, reduced joint damage, and risk minimization when patients engage in high-risk activities. Overall, it has given patients greater autonomy, independence, and flexibility of care. However, it has also meant that many children and adults with hemophilia no longer regularly report to the hospital, making it difficult for health care professionals to monitor the incidence of bleeding episodes, factor usage, and compliance with treatment regimens. Furthermore, it can result in delays in seeking appropriate medical care. 
The availability of mobile technology offers the potential to enhance, or at least to augment, the management of hemophilia in four principal ways by:

- Providing disease-specific information in a range of formats, including text, photo and video;

- Providing alerts to remind patients to take their treatment;

- Capturing user-entered data for stock control and payer purposes, and potentially providing instant guidance or treatment advice, whether on a patient-specific level or by comparison with a population (aggregated anonymized data held on remote servers - the "crowd in the cloud") in order to encourage positive health-related behaviors;

- Enhancing communication links between health care professionals and patients, and providing links to "approved" hemophilia-specific social networks.

\section{The shift to mobile}

Good record keeping is an essential component of homebased hemophilia care. Well-kept treatment diaries can help health care professionals to ensure optimal factor use, adherence with treatment regimens, and timely infusion practices. However, surveys have shown that record keeping habits among patients with hemophilia vary considerably according to personal preference and convenience. ${ }^{9}$

From the early days of home-based care, the hemophilia community has embraced electronic and software-based approaches to track factor use by patients. ${ }^{10-13}$ Many early attempts involved hand-held computers, which appeared to be an effective alternative to paper and pencil modes of data collection, being faster and preferred by most users. ${ }^{14}$ However, maintaining a separate device designed for frequent data collection is relatively intrusive and could never survive the growing adoption of the mobile phone. The mobile phone is now seen by many as an essential lifestyle accessory: phones are personal, unobtrusive, and almost always to hand. In our own focus group work, teenage boys have frequently told us that they check their phone before going to sleep at night and on waking each morning. The medical community has long recognized the potential of the mobile phone as a means of accessing patients and promoting positive health messages.

An Australian group recently undertook a one-year prospective study designed to assess the feasibility of using text messaging for the documentation of bleeds by children with severe hemophilia. ${ }^{15}$ Children (or their parents) were asked to maintain a bleed diary and received a weekly text message asking whether there had been a bleeding episode in the preceding week. The study achieved high response rates at minimal expense and intrusion, leading the authors to conclude that weekly text messaging offered a useful channel for the bidirectional transfer of information regarding bleeding rates, factor usage, and motivational messages to enhance treatment adherence.

\section{Enter the app}

Increasingly, treatment diaries for people with hemophilia have been developed as "apps." Many manufacturers of clotting factor products now offer patients the opportunity to monitor their treatment use and bleeds by means of promotional apps. Apps are available from Novo Nordisk Inc. (HemaGo; Plainsboro, NJ, USA), Pfizer, Inc. (HemMobile; New York, NY, USA), Bayer AG (FactorTrack; Leverkusen, Germany), Baxter (Beat Bleeds; Deerfield, IL, USA), and CSL Behring (HeliTrax; King of Prussia, PA, USA) among others. Most of these are well-developed apps with high production values that record factor usage regardless of hemophilia type or the type of medication used, reasons for an infusion, details about bleeding events and pain scores, and the personal impact of the bleeding episode. Most apps give patients the option of emailing their infusion and bleed history to themselves or their health care team. Some, such as Bayer's app, offer links to the company's range of educational tools and online resources. Some, such as Novo Nordisk's app, offer multiple profiles so that more than one family member can use the tool, which should help patients and their families track multiple medications and reminders for treatment or clinic appointments.

These apps are, of course, developed as marketing aids with commercial intent. While most of the companies claim they are unable to access patient-specific information, in most cases the company is able to see anonymized data. Data security is an important consideration with any health care app: how that data should be used by external parties is another.

Take-up of such company-sponsored apps is likely to be limited in countries such as Ireland and the UK, where product usage is tracked through a national database and where a central app is promoted to patients.

\section{Linking in with national databases}

In the past few years, the National Centre for Hereditary Coagulation Disorders in Dublin, Ireland, has implemented a mobile phone app as part of a track and trace system developed in response to the infection of hemophilia patients with HIV and hepatitis $\mathrm{C}$ from contaminated blood products supplied by the Blood Transfusion Service Board. In the 
early 2000s, the Lindsay Tribunal identified the need to be able to track and trace products safely through the supply chain, and subsequently such a system was developed with the international GS1 standards organization in collaboration with Dr Barry White, Ireland's National Director for Haemophilia Services.

At that time, patients were either collecting their medication from the hospital or were having it delivered by post or taxi. All product is now delivered through a scheduled delivery service by a cold-chain logistics company, and each package is labeled with a GS1 barcoding, a unique identifier that permits tracking and tracing of the product through the hospital and to the patient's home. A mobile phone app has been developed for the patients to download to their smart phone. The patient scans the barcode on the product packaging using their smart phone before self-medicating and the system verifies that the medication matches the prescription, is in date, and not on a recall list. The app also encourages the patient to enter critical information concerning the treatment. Usually this will be a scheduled prophylaxis or immune tolerance treatment. But if the patient indicates an "on demand" treatment then they are asked to indicate the location and severity of the bleed they wish to treat.

The app is database driven, enabling information to be managed and transmitted in real time. It is backed by a secure online portal that allows health care professionals to view data added by mobile users. Text or email alerts are used to prompt any action required as the result of data that exceed pre-defined thresholds.

The app is available on a range of smartphones and tablet devices, including those running Windows 8, Android, and the Apple iPhone and iPad. Importantly, they continue to function when mobile reception is lost temporarily, automatically updating the relevant database when reception is restored.

The app-based system can help the health care staff to identify cases where patients (or their carers) are overtreating, where patients are treating themselves at a time of day that is not appropriate to their lifestyle, or where patients are simply not taking their treatment as directed.

The app system may be used to schedule home visits by nurses and enables them to report on a range of patient checks and treatment via mobile devices. In this way it also gives managers and clinicians a complete view of nurse activity along with patient information, saving time in completing records. The system has changed the way that hemophilia nurses practice: less time spent in direct patient contact and more time spent in reviewing data submitted to the web application. This ability to access data in real time can be highly beneficial as it allows nurses to respond to patients with high risk bleeds much faster than if the information were submitted many months after the event, on paper and requiring input into a database system, where of course transcription errors may occur.

Staff at the National Centre for Hereditary Coagulation Disorders in Dublin are enthusiastic about the system, which they say has:

- Improved timeliness of medication administration

- Improved treatment compliance

- Reduced medication wastage

- Promoted patient empowerment

- Improved patient safety as product can be rapidly located should the need ever arise for a product recall.

The app-based system has been accepted by patients of all ages, most of whom prefer it to the older paper-based treatment diaries.

In the UK, patients who receive their prophylaxis treatment via home delivery companies are increasingly being urged to use Haemtrack, a secure online system developed by the UK's National Haemophilia Database. This allows patients to record the treatment batch numbers received and used. It functions as a treatment diary, offering patients access to a monthly calendar, where they can add their treatment and other details, including date and time, the product used, and the reason for treatment.

As of November 2013, some 1,800 patients in the UK were registered users of Haemtrack. ${ }^{16}$ However, the mobile app is only available to those with iPhones, effectively excluding those with Android-based devices. Furthermore, ensuring that patients enter their data accurately requires continual reinforcement from medical and nursing staff, and hemophilia centers vary considerably in the quality of the data submitted. Patients with an iPhone can input their data by means of an app available from the iPhone app store.

\section{Social benefits: the untapped potential of the app}

Collecting and using data on factor usage and bleeds is clearly of huge importance to clinicians and health care payers. But there can also be a huge benefit to patients where the feedback loop is closed and the data are used to provide more responsive care in acute situations. However, there is also great potential for combining hemophiliaspecific mobile phone-based apps with hemophilia-specific social networks, to provide the community and peer support that has been lacking since the development of home-based 
care. Such support is particularly needed as boys transition from pediatric services to adult services.

Historically, children with severe hemophilia spent considerable time in hospital and some even attended special schools. ${ }^{17}$ This allowed affected boys to build relationships with others also affected by hemophilia. Access to modern treatments now allows most adolescents to manage their condition at home. The transfer from the hospital to the home setting clearly offers many benefits, but it has resulted in fewer opportunities for children to meet and interact with peers facing the same health challenges. As a rare disease, it is unsurprising that several adolescents with hemophilia have alluded to feelings of social and geographic isolation in conversations with members of their comprehensive care team and during focus group discussions. ${ }^{18}$

Living with a long-term condition presents a significant source of stress and anxiety, particularly for people with hemophilia who must acquire considerable knowledge and management skills at a young age. ${ }^{19}$ Those who understand their disease and its treatment are typically less anxious and better able to manage their health both physically and emotionally. ${ }^{20}$ In addition to the health expertise provided by formal sources, patients living with long-term illness often depend on friends, relatives, and other patients for support and practical advice. This experiential knowledge may be lacking in adolescents with hemophilia, who now have less opportunity to interact with similarly affected peers, and particularly so for those who have no affected family members to approach for advice.

Both social networking and mobile phones are central features of most adolescents' lives and may offer health benefits to those with rare long-term health conditions by reducing isolation, enhancing access to peer support, and encouraging positive health choices. ${ }^{21,22}$ Social networks have the potential to provide an infrastructure that is currently lacking, to allow young people with hemophilia to overcome geographical and cultural barriers to information seeking and sharing and to restore the practical support networks that used to exist for this specific patient population.

While there are forums available for people with hemophilia on networks like Facebook, these tend to be open and often dominated by parents seeking support and advice. Most health care professionals avoid engaging with patients through these forums. Official guidance from the Nursing and Midwifery Council supports the responsible use of social networking sites by nurses, while reminding nurses of their duty to act responsibly at all times and uphold the reputation of the profession: inappropriate use of social networking sites, for instance by sharing confidential information online, will put the individual's registration at risk. ${ }^{23}$

We have explored the potential for a hemophilia-specific, restricted access social network through focus groups and surveys. In this work teenagers around the UK indicated that they would value the opportunity of being able to communicate with other teenagers with hemophilia for mutual advice. ${ }^{18}$ This, and the comments offered in our focus groups, represent an acknowledgment that while guidelines, health care professionals, and parents can all provide medical advice and support, adolescents with hemophilia face unique issues and functional limitations and would benefit from peer support via a social network from others who face (or have faced) similar challenges.

In response, the Haemnet charity has developed SixVibe (http://www.sixvibe.com), a secure online membershipbased community for young people with hemophilia and other bleeding disorders, with the aim of providing a supportive peer-to-peer environment as well as a source of disease-specific information developed specifically for the teenagers.

SixVibe is a professionally designed website, endorsed by the UK's Haemophilia Society and funded by medical educational grants from the pharmaceutical industry and donations from the charitable sector. Importantly, SixVibe is allied to Haemnet, a nurse-based professional network (http:// www.haemnet.com). This means that hemophilia nurses can refer patients to the site when they perceive that this would be beneficial to the patient. It also allows the site moderators to generate information for patients at the request of nurses, and to ensure the validity of those materials by "road testing" them on nurses before they are released to adolescents.

In the two years since it was established, SixVibe has attracted a substantial number of members, thus confirming the need for such a site. The challenge in maintaining a site for a small client group (the pool of teenagers with hemophilia in the UK is relatively small) is that the chance of somebody sitting at their computer and being active on the site when a new patient wishes to make contact is slim. Adding full mobile functionality to the site could allow us to overcome this limitation and help to develop the community to its full potential, such that patients are able to make contact with each other and provide support in real time. Furthermore, if we were able to pair the community with a data entry system such as Haemtrack, we could begin to build in elements of gamification - the use of game thinking and game mechanics in non-game contexts to engage users in solving problems. Game theory could be used not only to drive user engagement with both SixVibe and Haemtrack, but also to improve data quality, and to promote peer-to-peer support and 
learning through the development of a "buddy system" based on the training and development of expert patients.

\section{Conclusion}

Most health care professionals working in hemophilia recognize that good record-keeping is an essential component of home-based hemophilia care. We willingly add patients' data to national registries and databases. Too often, however, we use and interrogate those data for commercial and academic purposes, overlooking the fact that the data actually belong to our patients and should be used to improve the care of that individual. Patient-focused apps designed to respond to an individual's personal data may offer the potential to empower patients to live healthier lifestyles, reinforcing the care provided by health care professionals, offering a mechanism for peer-support, and promoting adherence to the individualized management plan. Furthermore, they could help to build the hemophilia community beyond the traditional patient advocacy group, which is particularly important for a rare disease.

\section{Disclosure}

The authors are the founders and owners of the SixVibe patient website. They report no other conflicts of interest in this work.

\section{References}

1. GSMA Intelligence. Global mobile penetration — subscribers versus connections [webpage on the Internet]. Available from: https://gsmaintelligence.com/analysis/2012/10/global-mobile-penetration-subscribersversus-connections/354/. Accessed April 8, 2014.

2. Free C, Phillips G, Galli L, et al. The Effectiveness of Mobile-Health Technology-Based Health Behaviour Change or Disease Management Interventions for Health Care Consumers: A Systematic Review. PLoS Med. 2013;10(1):e1001362.

3. IMS Institute for Healthcare Informatics. Patient Apps for Improved Healthcare: From Novelty to Mainstream. Pasippany, NJ: IMS Institute for Healthcare Informatics, 2013. Available from: http://www.imshealth. com/deployedfiles/imshealth/Global/Content/Corporate/IMS $\% 20$ Health\%20Institute/Reports/Patient_Apps/IIHI_Patient_Apps_Report. pdf. Accessed February 28, 2014.

4. US Department of Health and Human Services Food and Drug Administration. Mobile Medical Applications: Guidance for Industry and Food and Drug Administration Staff. Rockville, MD: US FDA; 2013. Available from: http://www.fda.gov/downloads/MedicalDevices/DeviceRegulationandGuidance/GuidanceDocuments/UCM263366.pdf. Accessed April 8, 2014.

5. Steinhubl SR, Muse ED, Topol EJ. Can mobile health technologies transform health care? JAMA. 2013;310(22):2395-2396.
6. Srivastava A, Brewer AK, Mauser-Bunschoten EP, et al; Treatment Guidelines Working Group The World Federation Of Hemophilia. Guidelines for the management of hemophilia. Haemophilia. 2013;19(1): e1-e47.

7. World Health Organization. Genomic resource centre: genes and human disease [webpage on the Internet]. Geneva: World Health Organization; 2014. Available from: http:/www.who.int/genomics/ public/geneticdiseases/en/index2.html\#Haemophilia. Accessed February 28, 2014.

8. Teital JM, Barnard D, Israels S, Lillicrap D, Poon MC, Sek K. Home management of haemophilia. Haemophilia. 2004;10(2):118-133.

9. Sholapur NS, Barty R, Wang G, Almonte T, Heddle NM. A survey of patients with haemophilia to understand how they track product used at home. Haemophilia. 2013;19(5):e289-e295.

10. Collins PW, Bolton-Maggs P, Stephenson D, Jenkins B, Loran C, Winter M. Pilot study of an Internet-based electronic patient treatment record and communication system for haemophilia, Advoy.com. Haemophilia. 2003;9(3):285-291.

11. Walker I, Sigouin C, Sek J, et al. Comparing hand-held computers and paper diaries for haemophilia home therapy: a randomized trial. Haemophilia. 2004;10(6):698-704.

12. Arnold E, Heddle N, Lane S, Sek J, Almonte T, Walker I. Handheld computers and paper diaries for documenting the use of factor concentrates used in haemophilia home therapy: a qualitative study. Haemophilia. 2005;11(3):216-226.

13. Mondorf W, Seigmund B, Manhel R, et al. Haemoassist - a hand-held electronic patient diary for haemophilia home care. Haemophilia. 2009;15(2):464-472.

14. Lane S, Heddle NM, Arnold E, Walker I. A review of randomized controlled trials comparing the effectiveness of hand held computers with paper methods for data collection. BMC Med Inform Decis Mak. 2006;6:23-33.

15. Broderick CR, Herbert RD, Latimer J, Mathieu E, van Doorn N, Curtin JA. Feasibility of short message service to document bleeding episodes in children with haemophilia. Haemophilia. 2012;18(6):906-910.

16. United Kingdom Haemophilia Centres Doctors' Organisation [homepage on the Internet]. Manchester: UKHCDO. Available from: http:// www.ukhcdo.org. Accessed April 8, 2014.

17. Aronstam A, Rainsford SG, Painter MJ. Patterns of bleeding in adolescents with severe haemophilia A. Br Med J. 1979;1(6161):469-470.

18. Khair K, Holland M, Carrington S. Social networking for adolescents with severe haemophilia. Haemophilia. 2012;18(3):e290-e296.

19. Civan A, McDonald DW, Unruh KT, Pratt W. Locating patient expertise in everyday life. GROUP ACM SIGCHI Int Conf Support Group Work. 2009;2009:291-300.

20. Barlow JH, Stapley J, Ellard DR, Gilchrist M. Information and self-management needs of people living with bleeding disorders: a survey. Haemophilia. 2007;13(3):264-270.

21. Stanton E, Lemer C. Networking for healthcare reform. J R Soc Med. 2010;103(9):345-346.

22. Valente TW. Network interventions. Science. 2012;337(6090):49-53.

23. Nursing and Midwifery Council. The code: standards of conduct, performance and ethics for nurses and midwives [webpage on the Internet]. NMC; 2008. Available from: http://www.nmc-uk. org/Publications/Standards/The-code/Introduction/. Accessed February 28, 2014.
Smart Homecare Technology and TeleHealth

\section{Publish your work in this journal}

Smart Homecare Technology and TeleHealth is an international, peer-reviewed, open access online journal publishing original research, reviews, editorials and commentaries on the application of technology to support people and patients at home and in assisted living centers to optimize healthcare and management resources. Specific topics in the journal include: Development and application of

\section{Dovepress}

devices within the home and embedded in appliances; Healthcare provider communication and education tools; and drug ordering and adherence. The manuscript management system is completely online and includes a very quick and fair peer-review system, which is all easy to use. Visit http://www.dovepress.com/ testimonials.php to read real quotes from published authors. 